\title{
Design and Implementation of an Educational Game Considering Issues for Visually Impaired People Inclusion
}

\author{
Luiz Valério Neto ${ }^{1}$, Joice L. Otsuka ${ }^{1}$ \\ ${ }^{1}$ Computing Department, Federal University of São Carlos (UFSCar) \\ São Carlos - SP - Brazil \\ luizvneto@gmail.com, joice@ufscar.br
}

\section{Summary}

In recent years, there has been an increase in researches focusing on the effectiveness of using videogames as educational digital resources that can contribute to the learning process at different levels, which has also subsidised the increase of the development of educational games. However, today's educational games are, in their vast majority, totally visual and not accessible to people with some types of visual impairment. In order to foster the use of modern educational resources in the classroom, such as digital games, it is important to ensure access to these resources for all. The scarcity of accessible software, which encompasses the growing use of educational games in schools, is due to the difficulties faced by designers and educators in adapting and/or developing technologies that allow integrated and complete access to students with visual impairment. The lack of funding and the insufficient knowledge about efficient design methods have been the major obstacles faced in the development of these technologies.

This work describes the process of design, implementation and evaluation of an accessible version for the educational game Em Busca do Santo Grau, following the EduGameAccess - a set of recommendation that integrates educational, playability and accessibility aspects for people with visual impairments. This is an applied, exploratory and qualitative research and the main procedures and methods conducted were: (i) a literature review on game accessibility; (ii) game design following EduGameAccess recommendations and the project requirements; (iii) incremental implementation interleaved with evaluations (inspections using EduGameAccess and user testing) and refinements.

As the main results, three accessible modules (or game levels) were developed: the Tutorial Level, the Football Pitch Level and the Library Level, each of the modules containing educational quests that can be solved by students with and without visual impairment. The user tests allowed to study and attest the effectiveness of the EduGameAccess recommendations in producing a fully accessible educational game, capable of providing students with equivalent experiences of fun and learning. The obstacles, lessons learnt, and results are also discussed [Valério Neto et al. 2019].

\section{References}

Valério Neto, L., Fontoura Junior, P. H. F., Bordini, R. A. and Otsuka, J. L. (2019). Design and Implementation of an Educational Game Considering Issues for Visually Impaired People Inclusion. In Proceedings of IEEE $19^{\text {th }}$ International Conference on Advanced Learning Technologies and Technology-enhanced Learning (ICALT 2019), Maceió - AL, Brazil, pp. 298302. doi: 10.1109/ICALT.2019.0009. 